\title{
Effect of drugs on carbohydrate metabolism
}

\section{By Vincent Marks, Department of Biochemistry, University of Surrey, Guildford $\mathrm{GU}_{2}{ }_{5} \mathrm{XH}$}

Many drugs affect carbohydrate metabolism in man. For some this is the action that is sought and the reason for their inclusion in the pharmacopoeia; for others it is an epiphenomenon or undesirable side-effect which may or may not be predictable.

Broadly speaking, drugs that affect carbohydrate metabolism can be divided (Table I) into those that tend: (a) to raise the fasting blood glucose concentration and reduce glucose tolerance, or (b) to lower the fasting blood glucose concentration, generally with little or no 'improvement' of glucose tolerance and, paradoxically, often the reverse. Occasionally, as in the instances of nicotinic acid and alcohol, the amount and the circumstances under which the drug is administered determine whether it produces a rise, a fall or no change in blood glucose concentration.

\section{Table $\mathrm{x}$. Drugs affecting glucose metabolism in man}

Predominantly hyperglycaemic

Polypeptide hormones* e.g. glucagon, growth hormone

Natural and synthetic glucocorticoids* e.g. cortisol, dexamethasone

Natural and synthetic catecholamines* e.g. adrenaline, isoprenaline

Oral contraceptives*

Caffeinet

Nicotinic acid $\uparrow$

Nicotine†

Narcotic analgesics e.g. morphine

Phenothiazines e.g. chlorpromazine

Diuretic benzothiadiazines and analogues e.g. chlorothiazide, chlorthalidone, frusemide

Non-diuretic benzothiadiazinest e.g. diazoxide

Probenecid

Phenytoin

Coumarins

*Hormones or analogues.

†'Dietary' constituents.

fAction intended.
Predominantly hypoglycaemic

Insulin*

Alcohol $\dagger$

Sulphonylureas * e.g. tolbutamide, chlorpropamide, glibenclamide

Biguanides* e.g. phenformin, metformin

Sulphonamides

Aspirin

Phenacetin

$\beta$-Adrenergic blockers e.g. propanolol

Monoamine oxidase inhibitors e.g. mebanazine

Phenylbutazone

Barbiturates 
Many of the most widely used 'drugs' that affect carbohydrate metabolism are either naturally occurring hormones or analogues of them; others are anutrient or even nutrient constituents of the diet, their effect depending as much upon their quantity and mode of administration as upon their molecular structure.

In this brief communication it is proposed to do no more than consider the mode of action, in so far as it is known, and effect upon glucose metabolism in man of some drugs mentioned in Table $\mathrm{I}$, selected largely on the basis of my personal interest. I have not considered the effects of drugs upon intermediary metabolism, or other carbohydrates of nutritional importance such as fructose, galactose and the disaccharides.

\section{Nicotinic acid}

This compound, best known as a vitamin, enjoys considerable popularity, particularly in the USA, when used in pharmacological doses as a hypolipidaemic agent in the treatment of essential hypercholesterolaemia, with or without associated hypertriglyceridaemia. Attempts have been made by the pharmaceutical industry to produce nicotinic acid derivatives which retain the hypolipidaemic effect but not its undesirable side-effects, which include unsightly and unpleasant flushing, itching, liver disease and impaired glucose tolerance (Gey \& Carlson, 197r).

One of the most intensively investigated nicotinic acid analogues is $\beta$-pyridyl carbinol (Ronicol). In man Ronicol is five or six times as potent in lowering fasting plasma cholesterol levels as is nicotinic acid, on a weight for weight basis, but unlike the native vitamin it is not hypoglyceridaemic. With small doses of Ronicol, such as are used for the treatment of chilblains and minor disorders of the peripheral vasculature, there is little discernable effect upon either glucose or lipid metabolism, but with larger doses it produces, like nicotinic acid itself, a rise in fasting blood glucose and moderate to severe impairment of glucose tolerance (Marks, Frizel, Twycross \& Buchanan, I971). The impairment of glucose tolerance is associated with plasma insulin hyper-responsiveness to both orally and intravenously administered glucose, and consequently is not due to suppression of pancreatic $\beta$-cell activity. Although the exact cause is still uncertain, current evidence suggests that glucose intolerance produced by prolonged nicotinic acid therapy is due to impaired peripheral utilization of glucose, possibly due to an increase in intracellular free fatty acids.

Curiously, in acute experiments, both nicotinic acid and Ronicol dramatically lower plasma free fatty acid levels by inhibiting adipose tissue lipolysis, reduce the blood glucose concentration and enhance peripheral glucose uptake. The improvement in glucose tolerance so produced is independent of insulin secretion and may be observed even in the totally insulinoprivic animal (Gross \& Carlson, I968). In diabetic subjects intravenous nicotinic acid has, by virtue of its ability to inhibit adipose tissue lipolysis, been used with advantage as an adjunct to insulin in the treatment of ketoacidotic pre-coma (Porte, $1969 ; \mathrm{R}$. Assan, personal communication). 


\section{Caffeine}

Caffeine, in addition to being an important constituent of the diet, is widely used either alone or in combination with other drugs as a therapeutic agent. It is, however, only within recent years that an effect upon carbohydrate and lipid metabolism has been recognized. Whilst several published series purport to demonstrate a hyperglycaemic effect of caffeine (Cheraskin \& Ringsdorf, 1968; Wachman, Hattner, George \& Bernstein, 1970) and impairment of glucose tolerance in man, others have revealed either no difference between caffeine-treated subjects and controls or actual improvement in glucose tolerance (Feinberg, Sandberg, De Castro \& Bellet, 1968; Daubresse, Luyckx, Demey-Ponsart, Franchimont \& Lefèbvre, 1973). Whilst it is difficult to reconcile these seemingly conflicting results, they are at least partially explicable on the basis of differences in experimental design, the dose of caffeine employed and choice of experimental subjects. Our own experience with moderate amounts of caffeine given as tea revealed no consistent effect upon oral glucose tolerance in normal individuals or those suffering from chemical diabetes. In certain individuals with chemical diabetes, however, caffeine produced improvement in glucose tolerance whilst in others it exacerbated the condition. This is not as strange as it appears at first sight. It is now well-recognized that caffeine inhibits intra- $\beta$-cell phosphodiesterase $(E C$ 3.I.4.I) and thereby enhances insulin release by adenyl cyclase-activating hormones, which probably includes those released by gut endocrine cells in response to the ingestion of glucose and other foods.

Daubresse et al. (1973) have recently shown that in normal subjects $250 \mathrm{mg}$ caffeine produces a small, but significant, over-all improvement in oral glucose tolerance without appreciable change in plasma insulin levels compared with controls, which led them to conclude that 'in normal subjects caffeine potentiates the insulinotropic effect of orally administered glucose'. These observations confirm the work of Feinberg et al. (1968) on the effect of caffeine on oral glucose tolerance, and contrast with the demonstration by Wachman et al. (1970) that caffeine impairs intravenous glucose tolerance in healthy human subjects.

These observations, coupled with others which implicate caffeine in alterations of carbohydrate and lipid metabolism (Cheraskin \& Ringsdorf, 1968; De Castro, Sandberg, Feinberg \& Bellet, 1969) and the pathogenesis of arteriovascular disease (Jick, Miettinen, Neff, Shapiro, Heinonen \& Slone, I973), point strongly to the need for further investigations into the metabolic as well as the cerebral stimulatory effects of this ubiquitous constituent of the normal diet.

\section{Diazoxide}

Diazoxide was first used experimentally for the treatment of hypertension, but was rapidly discarded for this purpose when its potent diabetogenic properties were recognized. Fortunately, its potential value for the treatment of hypoglycaemia was recognized by Drash \& Wolfe $(1964)$ and this accounts for its continued presence in the pharmacopoeia. 
Chemically diazoxide is a benzothiadiazine, to which generic family of drugs belong many of the most widely used oral diuretic agents. The diuretic benzothiadiazines, e.g. chlorothiazide, are themselves mildly diabetogenic but are so largely by virtue of their property of depleting body potassium (Seltzer \& Allen, 1969) though probably not exclusively so. Unlike diazoxide they do not inhibit insulin secretion, which is the main, but not sole, mechanism through which this agent and its analogues exert their hyperglycaemic effect. Hyperglycaemia and glucose intolerance produced by diazoxide remit spontaneously within $24-48 \mathrm{~h}$ of discontinuing the drug, and can be reversed immediately by treatment with hypoglycaemic sulphonylureas.

Nowadays diazoxide is the mainstay in the treatment of patients with spontaneous fasting hypoglycaemia unamenable to curative therapy (Marks, I972). Thus, an initially undesirable side-effect of diazoxide is now its main indication for use. Furthermore diazoxide, unlike other widely used benzothiadiazines, causes sodium retention and even oedema, which can be dispelled by adding a diuretic benzothiadiazine to the therapeutic regimen. This not only corrects electrolyte imbalance produced by diazoxide but enormously enhances its hyperglycaemic effect.

\section{Sulphonylureas}

Hyperglycaemia and glucose intolerance are encountered more frequently than spontaneous hypoglycaemia in clinical practice. Enormous research effort has, therefore, been devoted to the discovery and introduction into clinical practice of oral hypoglycaemic agents. Of those currently available the most important, both numerically and medically, are the sulphonylureas (Stowers, 1972), a family of drugs chemically related to, and developed from, the antimicrobial sulphonamides, a small number of which possess hypoglycaemic properties in their own right.

The sulphonylureas lower blood glucose levels mainly by sensitizing the $\beta$-cells of the pancreas to secrete insulin in response to the normal physiological stimuli, and, to a lesser extent, through other poorly understood mechanisms (Creutzfeldt, 1969). They can be divided into relatively short-acting drugs such as tolbutamide, tolazamide and glibenclamide, which are inactivated in the body by metabolic degradation, largely in the liver, and long-acting drugs, e.g. chlorpropamide, which are excreted virtually intact in the urine. As with insulin, overdosage with sulphonylureas can cause serious or fatal hypoglycaemia. This is particularly likely to occur in elderly, malnourished individuals, in whom detoxication mechanisms are slowed down and renal function is impaired.

Certain drugs, especially those which either (I) displace the sulphonylureas from their binding sites on plasma proteins, e.g. phenylbutazone, salicylate and warfarin, and thereby rapidly increase the amount of metabolically active sulphonylurea available to the tissues, or (2) compete with sulphonylureas for drug-metabolizing (mixed-function oxidase) enzymes in the liver, e.g. sulphaphenazol, dicoumarol and phenyramidole, and consequently decrease their rate of metabolic degradation, predispose to hypoglycaemia (Kristensen \& Christensen, I969). Ignorance, or neglect 
of possible drug inter-reactions is responsible for a large proportion of the fatal accidents attributable to sulphonylurea-induced hypoglycaemia, which differs from that induced by insulin in that it often persists for several days owing to retention of the offending drug in the body. Fortunately it is now possible to rapidly reverse the hypoglycaemic effect with diazoxide, even in instances in which large doses of sulphonylurea have been taken accidentally or with suicidal intent.

\section{Alcohol}

I would like to close this brief review of agents affecting carbohydrate metabolism in man by mentioning alcohol, which some would call a drug, both in its literal and its metaphorical sense, and others would consider a nutrient. In normal doses (i.e. sufficient to produce a blood alcohol level of less than $\mathrm{I} g / \mathrm{l}$ ) alcohol does not affect blood glucose levels. In larger, intoxicating amounts it produces in the recently-fed or overnight-fasted, alcohol-naive subject a rise in blood glucose concentration by activation of hepatic glycogenolysis (Marks, 1974). This effect, which is not observed in the alcohol-habituated subject, is mediated by the autonomic nervous system as well as by stimulation of glucagon and inhibition of insulin secretion.

Of much greater medical and social importance than this well-documented but inconsequential effect of alcohol upon blood glucose is its ability, even in nonintoxicating amounts, to produce severe, and occasionally fatal, hypoglycaemia both in alcohol-naive and habituated subjects (Marks \& Medd, I964). Under certain circumstances an alcohol-induced stupor or coma may merge insensibly and fatally into a hypoglycaemic coma. This may be one of the causes of death in subjects taken into police custody because of their disorderly behaviour and permitted to 'sleep it off' in the safety of a cell-only to be discovered dead next morning.

The main factor in the pathogenesis of alcohol-induced hypoglycaemia is inhibition of hepatic gluconeogenesis (Freinkel \& Arky, ig66). Consequently it is most often seen in situations where this is the sole or major source of glucose entering the blood, such as in overnight-fasted children or $3^{6-72} \mathrm{~h}$ acutely starved or chronically malnourished adults. It is probably for this reason that a disproportionately large number of the cases of alcohol-induced hypoglycaemia recorded in the literature relate to children who have deliberately or accidentally imbibed alcohol, or adults who live in parts of the world where malnutrition is common.

In the normal subject, alcohol seemingly does not interfere with the breakdown of glycogen pre-existing in the liver and its liberation into the blood as glucose, but there is evidence that it may potentiate the hypoglycaemic effect of exogenous insulin and other hypoglycaemic agents (Arky, Veverbrants \& Abramson, r968). Thus alcohol delays the restoration of blood glucose levels to normal during acute insulininduced hypoglycaemia, even in normal individuals. This may, in part, explain the particularly malign form of hypoglycaemia occasionally encountered in insulin- or drug-treated diabetics who have ingested large or even moderate amounts of alcohol.

In conclusion I would like to stress once again that a large and ever-growing number of drugs, quite apart from those used specifically for the purpose, can, and 
often do, have a profound effect upon carbohydrate metabolism in man. A detailed history, including reference to such homely remedies as aspirin and other, more rigidly controlled drugs, must be taken before a true assessment can be given of minor or even major abnormalities of glucose metabolism in either individual patients or groups of subjects.

\section{REFERENCES}

Arky, R. A., Veverbrants, E. \& Abramson, E. A. (x968). F. Am. med. Ass. 206, 575.

Cheraskin, E. \& Ringsdorf, W. M. Jr (1968). Lancet ii, 689 .

Creutzfeldt, W. (1969). In Pharmacokinetics and Mode of Action of Oral Hypoglycaemic Agents p. 201 [A. Loubatieres and A. E. Renold, editors]. Milano: Il Ponte.

Daubresse, J.-C., Luyckx, A., Demey-Ponsart, E., Franchimont, P. \& Lefèbvre, P. (1973). Acta diabetol. lat. ro, 1069.

De Castro, O. A. P., Sandberg, H., Feinberg, L. J. \& Bellet, S. (1969). Metabolism 18, 163.

Drash, A. \& Wolfe, F. (1964). Metabolism 13, 487 .

Feinberg, L. J., Sandberg, H., De Castro, O. \& Bellet, S. (rg68). Metabolism 17, 916.

Freinkel, N. \& Arky, R. A. (1966). Psychosom. Med. 28, 537.

Gey, K. F. \& Carlson, L. A. (editors) (1971). Metabolic Effects of Nicotinic Acid and its Derivatives. Bern: Hans Huber.

Gross, R. C. \& Carlson, L. A. (r968). Diabetes 17, 353 .

Jick, H., Miettinen, O. S., Neff, R. K., Shapiro, S., Heinonen, O. P. \& Slone, D. (1973). New Engl. Y. Med. 289, 63 .

Kristensen, M. \& Christensen, L. K. (1969). In Pharmacokinetics and Mode of Action of Oral Hypoglycaemic Agents p. II 6 [A. Loubatieres and A. E. Renold, editors]. Milano: Il Ponte.

Marks, V. (1972). In Hypoglycaemia and Diazoxide p. I53 [M. Austoni, editor]. Padova: Cedam.

Marks, V. (1974). Proc. R. Soc. Med. (In the Press.)

Marks, V., Frizel, D., Twycross, R. G. \& Buchanan, K. D. (197I). In Metabolic Effects of Nicotinic Acid and its Derivatives p. 96r [K. F. Gey and L. A. Carlson, editors]. Bern: Hans Huber.

Marks, V. \& Medd, W. E. (1964). F. ment. Sci. 110, 228.

Porte, D. Jr (1969). Archs intern. Med. r23, 252.

Seltzer, H. S. \& Allen, E. W. (1969). Diabetes 18, 19.

Stowers, J. M. (1972). Clins Endocr. Metab. x, 721.

Wachman, A., Hattner, R. S., George, B. \& Bernstein, D. S. (1970). Metabolism r9, 539. 